\section{Molecules and markers for endosonographers: What do we need to know and measure?}

\author{
M. Al-Haddad, M. B. Wallace \\ Division of Gastroentorology and Hepatology, Mayo Clinic \\ College of Medicine, Jacksonville, FL
}

\section{Introduction}

Endoscopic ultrasound (EUS) has become an indispensable tool for the diagnosis and staging for gastrointestinal and thoracic malignancies. Thanks to the safety and reliability of fine needle aspiration (FNA), this has become the method of choice for tissue acquisition from lymph nodes and tumors in various locations within the thoracic and abdominal cavities.

In the recent years, EUS-FNA has offered a better understanding of the pathophysiology of cancer. Similar to the adenoma-carcinoma sequence, other cancers including pancreas, lung, and esophagus may follow a comparable multi-step process of carcinogenesis. A necessary component of these is the ability to go "beyond cytology" to use fine needle aspirates to characterize the genomic and proteomic profiles of cancer and precancerous tissue.

\section{Molecular characterization of FNA samples: The basic concepts}

Although DNA is relatively stable in excised tissue, proteins and particularly RNA are unstable and must be handled in special ways to preserve them for characterization. The first role of the endoscopist is to obtain and preserve tissue suitable for further molecular characterization. In the first part of this review, we will focus on these initial steps. The methods for genomic and proteomic analyses are beyond the scope of this article and interested readers are referred to recent reviews [1,2].

RNA destroying-enzymes (RNAase enzymes) are very ubiquitous in the environment. Therefore, it is imperative to prevent rapid degradation of RNA in order to preserve fine needle aspirates for genomic studies. The simplest method to do this is through the use of RNAase inhibitor solutions such as RNA-later (Quagen Inc. Valencia Ca, USA) or RNA Stat 60 (RNA STAT-60 ${ }^{\circledR}$; TEL-TEST, Friendswood, TX, USA). The needle aspirate can be quickly mixed with these reagents. In our laboratory, we immediately place the FNA sample into an eppendorf tube in a wet ice bath, centrifuge in a small desktop device in the EUS room (1000 RPM at 4 deg. C, 1 minute), remove the supernatant, and resuspend the cell pellet in RNA stat 60. If RNA stabilization is not available on-site, the aspirate can also be snap frozen in liquid nitrogen and stored (preferably at -70 to $-80 \mathrm{C}$ ) for later RNA stabilization. Every ef-

Correspondence: Michael B. Wallace, M.D. · Associate Professor of Medicine . Division of Gastroenterology and Hepatology • Mayo Clinic Jacksonville 44500 San Pablo Rd · Jacksonville, FL 32224 ·E-mail: wallace.michael@mayo.edu

Bibliography: Endoscopy 2006; 38 (S1): S50-S53 (c) Georg Thieme Verlag KG Stuttgart · New York · ISSN 0013-726X · DOI 10.1055/s-2006-946652 fort should be made to handle the specimen in an RNAase-free environment including clean gloves and a surface that has been cleaned with an RNAase inhibitor solution.

In the remainder part of this review, we will focus on organspecific applications of the biological markers. This will include lesions of the pancreas and gastrointestinal stromal tumors (GIST). The use of biomarkers in staging non-small call lung cancer will be discussed in a separate review.

\section{Pancreatic lesions}

Diagnosis of early pancreatic cancer remains a challenge mainly due to the lack of a standard screening test. Therefore, improving long-term survival relies primarily on detecting and treating early pancreatic cancer and thus reducing the incidence of pancreatic cancer.

EUS is increasingly considered a valuable tool for the evaluation of the pancreatic cystic and solid lesions. It offers the highest resolution available for imaging the entire pancreatic parenchyma and duct. EUS provides detailed information regarding the wall and septations of pancreatic cysts and shed some light on the underlying pathology of such cysts.

Cystic lesions of the pancreas are being detected with increasing frequency, yet remain poorly understood. They are broadly divided into mucinous and nonmucinous lesions. Mucinous lesions (mucinous cystic neoplasms and intraductal papillary mucinous neoplasms (IPMNs)) are considered to be premalignant or malignant and surgical resection is generally recommended for appropriate candidates although it is increasing recognized that some IPMN lesions have a very low rate of malignant degeneration and may not require resection [3]. Non-mucinous lesions (serous cystadenomas and pseudocysts) have low or no malignant potential. Resection is only indicated in the setting of uncontrollable symptoms. Accurate methods to diagnose and determine the malignant potential of cystic lesions of the pancreas are highly desirable but current methods are limited. Currently, the only definite way to identify such lesions reliably is to surgically resect the part of the pancreas containing the suspicious lesions and examine them histopathologically.

EUS-guided fine needle aspiration (EUS-FNA) and cyst fluid analysis has been valuable to differentiate cystic lesions of the pancreas. As cytology of cyst fluid can be non-diagnostic due to low cellularity of the aspirated fluid, investigators have evaluated the use of tumor markers in the cyst fluid [4,5]. CEA and CA $72-4$ were found in high concentrations in mucinous cystic neoplasms. Various series have demonstrated that the cyst fluid CEA of benign cystic lesions is low [6,7]. The mean values ranged from 1.1 to $7.5 \mathrm{ng} / \mathrm{mL}$ in psuedocysts and serous cystadenomas.

The Cooperative Pancreatic Cyst Study evaluated the cystic fluid of 341 patients who underwent EUS-FNA of cystic lesions of the pancreas. Among these patients, 112 patients had surgical confirmation of the diagnosis. The receiver operator characteristic (ROC) curves were plotted for each tumor marker (CEA, CA 72 4, CA 125, CA 19-9, CA 15-3) to predict a mucinous or nonmu- 
cinous cystic lesion. The accuracy was greatest for CEA and CA $72-4$. The CEA cutoff value of $192 \mathrm{ng} / \mathrm{mL}$ provided the greatest accuracy of 0.79 (1.0 is perfect) with moderate sensitivity of 0.73 and specificity of 0.84 . For CA $72-4$, the cutoff value of 7 $\mathrm{ng} / \mathrm{mL}$ provided an accuracy of 0.72 , sensitivity of 0.80 and a specificity of 0.61 . This study confirmed that mucinous cystic lesions have a much higher concentration of CEA (mean CEA-5607 ng/ $\mathrm{mL}$ ) as compared to nonmucinous cystic lesions (mean CEA$284 \mathrm{ng} / \mathrm{mL})[8]$.

Microscopic premalignant lesions of the pancreas (e.g., IPMNs) are unlikely to be detected by serum markers given their noninvasive nature. No standard serum marker exists for screening of precancerous lesions, although these lesions pose the greatest risk for future invasiveness and, at the same time, have the highest chances of cure. It is projected that many of the underlying genetic abnormalities found in pancreatic ductal adenocarcinoma are found in such pre-cancerous lesions, although at lower prevalence. Therefore, some markers that are being investigated for pancreatic cancer diagnosis could potentially also predict the presence of these precancerous lesions [9].

Pancreatic juice analysis is being evaluated as a source of markers of pancreatic neoplasia [10], similar to other biomarker screening strategies (such as stool markers for colorectal neoplasia). Pancreatic juice can be collected during routine upper GI endoscopy after secretin stimulation. During EUS, fluid obtained from FNA of solid or cystic lesions represents an excellent source from which such markers can be obtained. This can be of particular importance in patients with diffuse and nonfocal changes of the pancreas as well as patients with a strong family history of pancreatic cancer. Pancreatic fluid contains higher concentrations of genomic and proteomic material allowing it to be a better substrate for further assays targeting screening for pancreatic cancer. For example, mutated $K$-ras, mutated p53 [11,12], and telomerase $[13,14]$, are more often detected in pancreatic secretions than in other sites such as plasma, duodenal fluid, or stool.

Mutant K-ras has been evaluated and found to be present in approximately $90 \%$ of pancreatic ductal adenocarcinomas. It can be easily detected using molecular assays and its presence has been shown to indicate worse prognosis [15]. However, K-ras mutations lack the specificity for pancreatic cancer; they were found to occur in smokers, patients with chronic pancreatitis, and pancreatic cystic lesions without cancer [16-18]. Mutant K-ras can also be detected in the blood of patients with advanced pancreatic cancer $[12,19]$. Despite this limitation, a sensitive assay has been recently developed that can quantify levels of mutant $K$-ras in blood and pancreatic juice. Further research is needed though to prove that quantifying mutant $K$-ras levels could improve the diagnostic utility of mutant K-ras [20].

TP53 gene mutations have been generally been investigated as a potentially specific diagnostic marker in a variety of cancers. Several technologically advanced assays have been developed (like single-strand conformational polymorphism) in an attempt to identify the entire spectrum of TP53 gene mutations. When detected in pancreatic ductal adenocarcinoma, it generally occurs late in the neoplastic process and indicates invasivness. These mutations were found in approximately $70 \%$ of invasive cancers
[21]. Although a few nucleotide hot spots of TP53 gene mutation are known to exist, mutations occur throughout the gene [22]. In one study, investigators reported the presence of TP53 gene mutations in pancreatic juice samples and in brush cytology specimens of $40 \%-50 \%$ of patients with pancreatic cancers [23]. Therefore, the detection of P53 mutations in pancreatic juice has the potential to become an applicable diagnostic strategy if improvements in mutation detection technology can enable accurate detection of such mutations.

The detection and quantification of aberrantly methylated DNA in pancreatic fluid can be a promising approach to the diagnosis of pancreatic cancer. A recent study by Matsubayashi et al evaluated the presence of DNA methylation alterations in the pancreatic juice of patients with suspected pancreatic disease [10]. The prevalence of pancreatic juice methylation in patients with chronic pancreatitis was less than in patients with pancreatic cancer but higher than in controls.

Finally, combining histological examination and immunoassays can significantly enhance diagnostic accuracy of pancreatic cancer. In a recent study from Japan, a total of 62 patients with solid pancreatic masses (51 pancreatic cancers and 11 chronic pancreatitis) underwent EUS-FNA [24]. Tissue specimens were evaluated by routine histological examination and p53 immunostaining. p53 protein overexpression was observed in $67 \%$ patients with pancreatic cancer, but not in patients with chronic pancreatitis.

\section{Gastrointestinal Stromal Tumors (GIST)}

Advances in immunohistochemical analysis changed the way gastrointestinal myogenic tumors are classified. Currently, the term "gastrointestinal stromal tumor" (GIST) is used to designate the largest category of primary nonepithelial neoplasms of GI lesions [25-28]. EUS has become a well-established technique for the diagnosis and follow-up of this group of gastrointestinal pathologies. It provides detailed images of GI wall structure and indicates the layer of origin of submucosal tumors. For example, a diagnosis of myogenic tumor can be made confidently when a submucosal tumor is seen to arise from the fourth hypoechoic sonographic layer (muscularis propria) of the adjacent normal GI tract wall. However, the differentiation between benign and malignant myogenic tumors can be endosonographically difficult. Several criteria have been suggested to indicate malignancy including size $(>3 \mathrm{~cm})$, irregular border and the presence of internal cystic spaces $[25,29,30]$. Surgical resection has classically been recommended for those lesions with suspicious features. Standard endoscopic biopsies typically do not help in the diagnosis of submucosal lesions.

When compared to lymph nodes and pancreatic masses, EUSFNA is reported to be less useful for the histopathologic diagnosis of submucosal tumors [31,32]. However, if an adequate sample can be obtained, it is possible to count mitotic figures, the most significant indicator of biologic behavior $[33,34]$. By using the EUS-FNA technique, adequate specimens are usually obtained for histology and differential immunophenotyping of GISTs. With advances in immunohistochemistry, several investigators 
have reported that most myogenic tumors express CD34 and a growth factor receptor with tyrosine kinase activity termed KIT. This receptor, the product of the proto-oncogene c-kit (located on chromosome 4q11-q12), can be detected by immunohistochemical staining for CD117. All GISTs are immunohistochemically positive for KIT (CD117) $[27,28,25]$. Although many other tumor types could be CD117 positive, most of them do not occur in the gastrointestinal tract. Positive CD117 staining (and in most cases positive CD34 staining) in the appropriate clinical setting, with compatible gross and microscopic findings, are the defining features of these tumors [36]. Moreover, tumor immunophenotype is reported to strongly reflect histologic behavior of GISTs $[37,38]$. For example, Newman et al [39] reported that tumors with a neural phenotype have the best prognosis. However, the results of these studies were based on analysis of surgically resected lesions.

As with pancreatic lesions, the importance of combining EUS and pathology has been emphasized. In a study by Nobuhiro et al [40], preoperative EUS alone and in combination with histopathology/immunohistochemistry was evaluated. The overall accuracy for the diagnosis of malignant GIST was $78 \%$ by EUS imaging alone and $91 \%$ by histopathologic evaluation (H\&E staining) of specimens obtained by EUS-FNA. Immunohistochemical expressions of several markers including c-kit, CD34, muscle actin, S-100 were evaluated on EUS-FNA and post surgical resection specimens. For the diagnosis of malignant GIST, the accuracy, sensitivity, and specificity of EUS-FNA with the addition of Ki-67 immunohistochemical staining were $100 \%$. Further studies are needed to confirm this finding and reevaluate the rest of the markers.

\section{Conclusion}

EUS plays an important role in the diagnosis of GI cancers. It has helped shift the current practice from simple staging to early detection and treatment. The recent scientific leaps in the recognition of genetic markers and phenotypic molecular expressions have largely contributed to this shift. The best approach for early cancer detection seems to rely on the coupling of EUS-FNA and available gene markers and immunoassays.

\section{References}

${ }^{1}$ Ilyin SE, Belkowski SM, Plata-Salaman CR. Biomarker discovery and validation: technologies and integrative approaches. Trends Biotechnol 2004; 22 (8): $411-416$

2 Pritzker KP, Azad A. Genomic biomarkers for cancer assessment: implementation challenges for laboratory practice. Clin Biochem 2004; 37 (7): $642-646$

${ }^{3}$ Brugge WR. Approach to cystic pancreatic lesions. Gastrointest Endosc Clin N Am 2005; 15 (3): 485-496, viii

${ }^{4}$ Khalid A et al. Use of microsatellite marker loss of heterozygosity in accurate diagnosis of pancreaticobiliary malignancy from brush cytology samples. Gut 2004; 53 (12): 1860 - 1865

${ }^{5}$ McCarthy DM et al. Novel markers of pancreatic adenocarcinoma in fine-needle aspiration: mesothelin and prostate stem cell antigen labeling increases accuracy in cytologically borderline cases. Appl Immunohistochem Mol Morphol 2003; 11 (3): 238-243

${ }^{6}$ Frossard JL et al. Performance of endosonography-guided fine needle aspiration and biopsy in the diagnosis of pancreatic cystic lesions. Am J Gastroenterol 2003; 98 (7): 1516-1524
${ }^{7}$ Hammel P et al. Diagnostic value of CA $72-4$ and carcinoembryonic antigen determination in the fluid of pancreatic cystic lesions. Eur J Gastroenterol Hepatol 1998; 10 (4): 345 - 348

${ }^{8}$ Brugge WR et al. Diagnosis of pancreatic cystic neoplasms: a report of the cooperative pancreatic cyst study. Gastroenterology 2004; 126 (4): $1330-1336$

${ }^{9}$ Goggins M. Molecular markers of early pancreatic cancer. J Clin Oncol 2005; 23 (20): $4524-4531$

${ }^{10}$ Matsubayashi $\mathrm{H}$ et al. DNA methylation alterations in the pancreatic juice of patients with suspected pancreatic disease. Cancer Res 2006; 66 (2): $1208-1217$

${ }^{11}$ Wilentz RE et al. K-ras mutations in the duodenal fluid of patients with pancreatic carcinoma. Cancer 1998; 82 (1): 96-103

12 Yamada T et al. Detection of K-ras gene mutations in plasma DNA of patients with pancreatic adenocarcinoma: correlation with clinicopathological features. Clin Cancer Res 1998; 4 (6): 1527-1532

13 Ohuchida $\mathrm{K}$ et al. A highly sensitive and quantitative telomerase activity assay with pancreatic juice is useful for diagnosis of pancreatic carcinoma without problems due to polymerase chain reaction inhibitors: analysis of 100 samples of pancreatic juice from consecutive patients. Cancer 2004; 101 (10): 2309-2317

${ }^{14}$ Myung SJ et al. Telomerase activity in pure pancreatic juice for the diagnosis of pancreatic cancer may be complementary to K-ras mutation. Gastrointest Endosc 2000; 51 (6): 708 - 713

${ }^{15}$ Brunner TB et al. Pancreatic cancer cell radiation survival and prenyltransferase inhibition: the role of K-Ras. Cancer Res 2005; 65 (18): $8433-8441$

${ }^{16}$ Caldas $\mathrm{C}$ et al. Frequent somatic mutations and homozygous deletions of the p16 (MTS1) gene in pancreatic adenocarcinoma. Nat Genet 1994; 8 (1): $27-32$

${ }^{17}$ Kalthoff $\mathrm{H}$ et al. p53 and K-RAS alterations in pancreatic epithelial cell lesions. Oncogene 1993; 8 (2): 289-298

18 Tada $\mathrm{M}$ et al. Detection of ras gene mutations in pancreatic juice and peripheral blood of patients with pancreatic adenocarcinoma. Cancer Res 1993; 53 (11): 2472-2474

${ }^{19}$ Mulcahy HE et al. A prospective study of K-ras mutations in the plasma of pancreatic cancer patients. Clin Cancer Res 1998; 4 (2): 271 - 275

${ }^{20}$ Shi $\mathrm{C}$ et al. LigAmp for sensitive detection of single-nucleotide differences. Nat Methods 2004; 1 (2): 141 - 147

${ }^{21}$ Caldas $\mathrm{C}$ et al. Detection of K-ras mutations in the stool of patients with pancreatic adenocarcinoma and pancreatic ductal hyperplasia. Cancer Res 1994; 54 (13): 3568 - 3573

${ }^{22}$ Hollstein M et al. p53 mutations in human cancers. Science 1991; 253 (5015): 49-53

${ }^{23}$ Sturm PD et al. The potential diagnostic use of K-ras codon 12 and p53 alterations in brush cytology from the pancreatic head region. J Pathol 1998; 186 (3): $247-253$

${ }^{24}$ Itoi T et al. Immunohistochemical analysis of p53 and MIB-1 in tissue specimens obtained from endoscopic ultrasonography-guided fine needle aspiration biopsy for the diagnosis of solid pancreatic masses. Oncol Rep 2005; 13 (2): 229-234

${ }^{25}$ Franquemont DW. Differentiation and risk assessment of gastrointestinal stromal tumors. Am J Clin Pathol 1995; 103 (1): 41 - 47

${ }^{26}$ Rosch T et al. Endosonographic diagnosis of submucosal upper gastrointestinal tract tumors. Scand J Gastroenterol 1992; 27 (1): 1 - 8

${ }^{27}$ Miettinen M, Virolainen M, Maarit Sarlomo R. Gastrointestinal stromal tumors-value of CD34 antigen in their identification and separation from true leiomyomas and schwannomas. Am J Surg Pathol 1995; 19 (2): 207-216

${ }^{28}$ Kindblom LG et al. Gastrointestinal pacemaker cell tumor (GIPACT): gastrointestinal stromal tumors show phenotypic characteristics of the interstitial cells of Cajal. Am J Pathol 1998; 152 (5): 1259-1269

${ }^{29}$ Boyce GA et al. Evaluation of submucosal upper gastrointestinal tract lesions by endoscopic ultrasound. Gastrointest Endosc 1991; 37 (4): 449-454

${ }^{30}$ DeMatteo RP et al. Two hundred gastrointestinal stromal tumors: recurrence patterns and prognostic factors for survival. Ann Surg 2000; 231 (1): $51-58$

${ }^{31}$ Wiersema MJ et al. Endosonography-guided fine-needle aspiration biopsy: diagnostic accuracy and complication assessment. Gastroenterology 1997; 112 (4): 1087 - 1095

32 Matsui $\mathrm{M}$ et al. Preliminary results of fine needle aspiration biopsy histology in upper gastrointestinal submucosal tumors. Endoscopy 1998; 30 (9): $750-755$ 
${ }^{33}$ Amin MB et al. Prognostic value of proliferating cell nuclear antigen index in gastric stromal tumors. Correlation with mitotic count and clinical outcome. Am J Clin Pathol 1993; 100 (4): 428-432

${ }^{34}$ Carrillo $\mathrm{R}$ et al. Prognostic significance of DNA ploidy and proliferative index (MIB-1 index) in gastrointestinal stromal tumors. Hum Pathol 1997; 28 (2): $160-165$

${ }^{35}$ Sarlomo-Rikala M et al. CD117: a sensitive marker for gastrointestinal stromal tumors that is more specific than CD34. Mod Pathol 1998; 11 (8): $728-734$

${ }^{36}$ Graadt van Roggen JF, van Velthuysen ML, Hogendoorn PC. The histopathological differential diagnosis of gastrointestinal stromal tumours. J Clin Pathol 2001; 54 (2): 96-102

37 Ueyama $\mathrm{T}$ et al. A clinicopathologic and immunohistochemical study of gastrointestinal stromal tumors. Cancer 1992; 69 (4): 947-955

${ }^{38}$ Seidal T, Edvardsson H. Expression of c-kit (CD117) and Ki67 provides information about the possible cell of origin and clinical course of gastrointestinal stromal tumours. Histopathology 1999; 34 (5): 416 - 424

${ }^{39}$ Newman PL, Wadden C, Fletcher CD. Gastrointestinal stromal tumours: correlation of immunophenotype with clinicopathological features. J Pathol 1991; 164: 107 (2)- 117

${ }^{40}$ Ando $\mathrm{N}$ et al. The diagnosis of GI stromal tumors with EUS-guided fine needle aspiration with immunohistochemical analysis. Gastrointest Endosc $2002 ; 55$ (1): $37-43$ 